\title{
Linear and nonlinear tunable optical properties of intersubband transitions in GaN/AlN quantum dots in presence and absence of wetting layer
}

\author{
A. Khaledi-Nasab \\ ali.khaledi1989@gmail.com \\ M. Sabaeian
}

M. Rezaie

\section{Mohammad-Rezaee}

Department of Physics, Faculty of Science, Shahid Chamran University of Ahvaz, Ahvaz, Iran

Department of Physics, Faculty of Science, Shahid Chamran University of Ahvaz, Ahvaz, Iran

Department of Physics, Faculty of Science, Shahid Chamran University of Ahvaz, Ahvaz, Iran

In this study we have performed a numerical approach to investigate the optical properties of GaN/AIN quantum dots (QDS). We have used nice homemade finite element method (FEM) codes to solve the Schrödinger equation, in presence and absence of wetting layer. The optical properties of both well-known, truncated pyramids-shaped, wurtize (WZ) and zinc blande (ZB) QDs have been investigated. It is demonstrated, there is slight amount of difference between all orders of absorption coefficients and relative refractive index changes (RRIC) for both structures. The effect of relaxation rate studied as well. Overlay it is shown that the optical properties ZB/WZ QDS could be engineered in well-manner.

[DOI: http://dx.doi.org/10.2971/jeos.2014.14011]

Keywords: GaN/AIN quantum dot, wurtize, Zinc blende, linear and nonlinear optical properties

\section{INTRODUCTION}

During the last few years, semiconductor QD heterostructures have been subject of extensive interest due to their advantages over quantum wells and wires, ultimate three-dimensional (3D) confinement structures of carriers, promising efficient lasers and optical amplifiers as well as possible applications for memory storage and quantum computing [1]. Owning to the lattice mismatch between layers and the base material, the strain effect drives the QDs towards 3D islands. The unconverted QD material is called wetting layer [2]. Semiconductor QDs are potentially ideal for achieving large arrays of reproducible entangled photon generators as they allow the generation of requested photons $[3,4]$ with no dependence to probabilistic processes [5]. Recently, in state of the art treatise it is reported that QDs arrays can be employed to obtain areas with up to $15 \%$ polarization entangled photon emitter along with fidelities as high as $0.721 \pm 0.043$ [6]. Moreover, a record source efficiency of 0.72 , combined with pure single-photon emission is demonstrated by J. Cluden et al. [7] by making use of a QD in a photonic nanowire under optical pumping which it would be potentially a high efficient QD based single photon source. E. Stinaff et al. [8] reported that asymmetric pair of InAs QDs tuned into resonance by applying an electrical field which it leads to the formation of a coherent molecular wavefunction by a single hole. Also they have found that coulomb interactions shift the molecular resonance of charged exciton with respect to the single charge, enabling the QDs lightinduced coupling. Theoretically, Michael et al. [9] explored the slowdown of optical pulses due to the quantum coherence effects for an active material consisting of double InGaAs-based
QD molecules. Also the dependencies of slowdown, the shape of propagating probe pulses on lattice temperature and drive intensities have been explored as well. Ramanathan et al. [10] have conducted an experimental research on the effect of tunnel coupling on the Quantum-Confined Stark Effect for excitons in the coupled InAs/GaAs QDs, precisely they demonstrated that any decrease of dots' separation by the barrier leads to a remarkable escalation on zero-field dipole moments and polarizability. The transmission coefficient and group index properties of the probe field in a four-level QD molecule is being investigated in literature where the author found that the tunnel coupling plays a very important role in realizing the transmission coefficient of the probe field [11]. Moreover the Second harmonic generation and optical rectification [12] as well as the impact of Woods-Saxon potential on InAs/GaAs QDs [13] is explored as well.

Particularly, group III-nitride materials are very appropriate for implementation in high-power, high frequency, and hightemperature electronics, to mention but a few [14]. It is worth mentioning that these materials could be crystallize either with wurtzite (WZ) crystal structure which is a thermodynamically stable configuration or with zinc-blende (ZB) structure which is modified meta-stably [15].

It is shown that under particular growth conditions, the strain relaxation of $\mathrm{WZ}$ GaN grown by $\mathrm{MBE}$ on $\mathrm{AlN}$ occurs through $3 \mathrm{D}$ islanding after deposition of a few 2D monolayers. The characteristics of this growth mode are known as Stranski- 
Krastanov (SK). In this mode the deposition of a strained wetting layer is followed by elastic relaxation through 3D islanding which results in free surface formation [16]. So far various materials have been developed by SK under compressive stress, such as InAs /GaAs [17], InP/GaInP [18] or SiGe/Si [19]. In addition, single polar GaN/AIN QDs have been fabricated by metal-organic chemical vapor deposition (MOCVD) method [20]. Indeed, GaN/AIN QDs with wide band gap have attracted remarkable attention for potential application in optical, optoelectronic, and electronic devices [21]; such as developing single electron transistors [22], ultraviolet sources [23] as well as detectors [24]. More accurately, GaN and AIN form a continuous alloy system whose direct band-gaps exhibit wide profile (3.4 and $6.2 \mathrm{eV}$ [25]-[27], respectively) and owning to low dielectric constants and large effective masses, consistently present strong excitonic binding energies $(26 \mathrm{meV}$ for $\mathrm{GaN}$ ) [28]. The lattice mismatch is about $2.4 \%$ between GaN and AlN [29].

P. Lefebvre et al. [28] illustrated that in the grown GaN/AIN QDs along the (0001) axis in the WZ phase, the optical characteristics are dominated by huge on-axis internal electric fields, leading to a very low oscillator strength and complex dynamical behavior. Moreover, The cathodoluminescence investigations of GaN/AlN QD optical emissions in order to assess the reliability of the depth-resolved cathodoluminescence technique in revealing QD-related optical emissions as well as to distinguish between layers with different nominal GaN coverage's were reported by Salviati et al. [30]. A microphotoluminescence study of single GaN/AIN QDs in nanowires is presented by Renard et al. [31] and they observed low excitation power, single exciton lines with full width at half-maximum as narrow as $1 \mathrm{meV}$. Also it is demonstrated that investigating the excitation power dependence of the emission give permission to identify the biexciton transitions. V. A. Fonoberov et al. [32] have worked both theoretically and experimentally on the excitonic optical properties as well as have found the discrepancies between the radiative lifetime for different sizes of WZ and ZB QDs. N. Skoulidis et al. have studied the wavefunctions and the oscillator strength both close to the fundamental gap [33].

In this paper we will report the optical properties of both truncated cone shaped WZ and ZB GaN/AlN QDs. Indeed, the linear, second and third order nonlinear susceptibilities will be employed to investigate the linear and nonlinear absorption coefficient as well as relative refractive index changes both in the presence and absence of wetting layer. The strain energy in QDs structure can affect the energy eigenvalues, envelope functions, therefore it will profound influence on the optical properties of QDs. Nevertheless the strain effects can be compensated using strain compensation layers as demonstrated by Nuntawong et al. [34, 35] The strain effect has also been compensated by Zhao et al. [36] and Park et al. [37] in InGaN/AlGaN and InGaN/InGaN quantum wells, respectively. Therefore we avoid considering the strain in our computational experiments based on mentioned references.

\section{MODEL AND EQUATIONS}

In our modeling, confined electrons on the self-assembled GaN/AlN QD structures with and without wetting layers are examined based on the $\vec{k} \vec{p}$ method. In this case we will use the presented mythology in reference [2] as well used by reference [38]. This model illustrates interpretations for states with arbitrary kinetic energy in the quantum well (QW) wetting layer. States of electrons for single QD/QW wetting layer system will asymptotically approach either 'pure' QW states far from the QD, or zero if the state is a 'true' state of QD [2].

In the following, a model procedure will be presented that allows us to determine energy eigenvalue and associated wavefunction of any cylindrically symmetric QDs structure in presence/absence of wetting layers. The starting point is the 1band Schrödinger equation in the effective mass approximation:

$$
-\frac{\hbar^{2}}{2} \nabla\left(\frac{1}{m_{e}(\vec{x})} \nabla \psi(\vec{x})\right)+V_{e}(\vec{x}) \psi(\vec{x})=E \psi(\vec{x}),
$$

Where $\hbar, m_{e}(\vec{x}), V_{e}(\vec{x}), E$ and $\psi(\vec{x})$ are Planck's constant divided by $2 \pi$, the electron effective mass, the band-edge potential energy, the energy eigenvalue and electron envelope function, respectively. According to the assumption that we have cylindrical symmetry so $\vec{x}=(z, r \phi), V_{e}(\vec{x})=V_{e}(z r)$, and $m_{e}(\vec{x})=m_{e}(z, r)$. Where $z$ and $r$ are the axial and radial coordinates, and $\phi$ is the azimuthal angle ranging from $0-2 \pi$. Cylindrical symmetry allows us to use the separation of variables technique as follows for the envelop function:

$$
\psi(\vec{x})=\chi(z, r) \Phi(\phi),
$$

By using some simple and straight forward mathematical treatment Eq. (1) becomes:

$$
\begin{aligned}
& \frac{-\hbar^{2}}{2} \frac{\partial}{\partial z}\left(\frac{1}{m_{e}(z, r)} \frac{\partial \chi(z, r)}{\partial z}\right) \Phi(\phi) \\
- & \frac{\hbar^{2}}{2 r} \frac{\partial}{\partial r}\left(\frac{r}{m_{e}(z, r)} \frac{\partial \chi(z, r)}{\partial r}\right) \Phi(\phi) \\
& -\frac{\hbar^{2} \chi(z, r)}{2 m_{e}(z, r) r^{2}} \frac{\partial^{2} \Phi}{\partial \phi^{2}}+V_{e}(z, r) \chi(z, r) \Phi(\phi) \\
= & E \chi(z, r) \Phi(\phi)
\end{aligned}
$$

For the aim of simplification we divide both side of Eq. (3) by $\frac{\Phi(\phi) \chi(z, r)}{m_{e}(z, r) r^{2}}$, therefore Eq. (3) can be rearrange as:

$$
\begin{aligned}
& \frac{m_{e}(z, r) r^{2}}{\chi(z, r)}\left(\frac{-\hbar^{2}}{2} \frac{\partial}{\partial z}\left(\frac{1}{m_{e}(z, r)} \frac{\partial \chi(z, r)}{\partial z}\right)\right. \\
& \left.-\frac{\hbar^{2}}{2 r} \frac{\partial}{\partial r}\left(\frac{r}{m_{e}(z, r)} \frac{\partial \chi(z, r)}{\partial r}\right)\right) \\
& +m_{e}(z, r) r^{2}\left(V_{e}(z, r)-E\right) \\
& =-\frac{\hbar^{2}}{2} \frac{\partial^{2} \Phi}{\partial \phi^{2}} \frac{1}{\Phi(p h i)} \equiv-\frac{\hbar^{2}}{2} l^{2}
\end{aligned}
$$

An immediate solution for last part of Eq. (4) could be proposed as:

$$
\Phi(\phi)=e^{i l \phi},
$$


Where $l$ is a separation constant. As the envelope function must be single-valued under $2 \pi$ rotation. In our modeling $l$ can takes values of $l=0, \pm 1, \pm 2, \ldots$.

$$
\begin{aligned}
& \frac{-\hbar^{2}}{2} \frac{\partial}{\partial z}\left(\frac{1}{m_{e}(z, r)} \frac{\partial \chi_{l}}{\partial z}\right)-\frac{\hbar^{2}}{2 r} \frac{\partial}{\partial r}\left(\frac{r}{m_{e}(z, r)} \frac{\partial \chi_{l}}{\partial r}\right) \\
& +\left(\frac{\hbar^{2} l^{2}}{2 m_{e}(z, r) r^{2}}+V_{e}(z, r)\right) \chi_{l}=E \chi_{l}
\end{aligned}
$$

Eq. (6) has the geenral form of:

$$
\nabla\left(-c \nabla \chi_{l}\right)+\alpha \chi_{l}+\beta \nabla \chi_{l}=E \chi_{l}
$$

With $\beta=-\frac{\hbar^{2}}{2 m_{e}} \frac{1}{r}, \alpha=\frac{4 \hbar^{2} l^{2}}{m_{e} r^{2}}+V$, and $c=\frac{\hbar^{2}}{2 m_{e}}$.

Both original and simplified forms of Schrödinger equation, contain discontinious coefficients under spatial derivative operatros, owing to different material charactrsitcs of GaN and AlN. Physically, in the modeling procedure the following boundary condition must be satisfied.

$$
\begin{aligned}
& \psi \in C(Q), \text { and }\left(-\frac{1}{m_{e} \nabla \psi(\vec{x})} \cdot \vec{n}\right)_{Q D / \text { wettinglayer }} \\
= & \left(-\frac{1}{m_{e} \nabla \psi(\vec{x})} \cdot \vec{n}\right)_{\text {matrixes }}
\end{aligned}
$$

Which $m_{e}$ is the electron effective mass which has specific values for every material, $n$ is the outward normal vector, $C$ is the class of continuous function, $Q$ is the spatial $(z, r)$ domain of interest. The effective mass potential is considered to be constant for every material. The envelope function solution for a stepwise constant effective mass potential is:

$$
\psi_{n k}(z, r, \phi)=A_{n k} Z_{n k}(z) J_{n}(k r) e^{i l \phi}
$$

$Z_{n k}$ could be obtained from the solution of

$$
={ }_{=} \frac{\partial}{\partial z}\left(\frac{1}{m_{e}(z)} \frac{\partial Z_{n k}}{\partial z}\right)+\frac{2}{\hbar^{2}}\left(E-v_{e}(z)-\frac{\hbar^{2} k^{2}}{2 m_{e}(z)}\right) Z_{n k}
$$

$\psi_{n k}$ must satisfy the following condition:

$$
\begin{cases}\frac{\partial \psi_{n k}}{\partial r}-\frac{K\left(J_{n-1}(k r)-J_{n+1}(k r)\right)}{2 J_{n}(k r)} \psi_{n k}=0, & \text { if } J_{n}(k r) \neq 0 \\ \psi_{n k}=0, & \text { if } J_{n}=0\end{cases}
$$

Inasmuch as

$$
J_{n-1}(y)-J_{n+1}(y)=\frac{2 \partial J_{n}(y)}{\partial y}
$$

Prior to proposing a solution for simplified Schrödinger equation, appropriate boundary conditions must be supplemented. In order to employ adequate boundary conditions we consider the $z$ axis to be perpendicular to wetting layer plane also $r$ axis is chosen to be on wetting layer plane (Figure 1). As far as we are interested in the confined electronic states, the Dirichlet boundary condition $(\psi=0)$ is imposed for boundaries 1 and 3. In order to satisfy Eq. (8) for the interior boundaries, the continuity condition

$$
\left(-\frac{1}{m_{e} \nabla \psi(\vec{x})} \cdot \vec{n}\right)_{G a N}=\left(-\frac{1}{m_{e} \nabla \psi(\vec{x})} \cdot \vec{n}\right)_{A l N}
$$

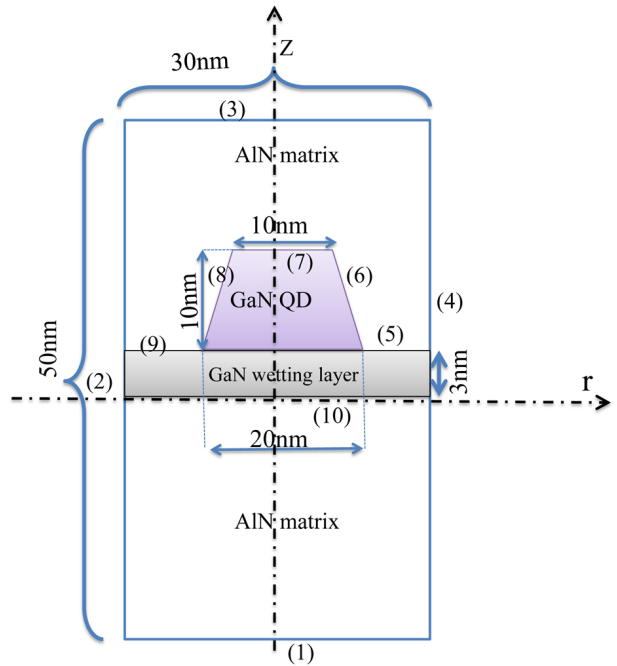

FIG. 1 The Geometry of and numbered boundary of GaN/AIN QDS.

is applied for boundaries 5, 6, 7, 8, 9, and 10. For relatively large values of $r$, the envelope function for GaN/AIN QDs approaches asymptotically either the envelope function for the pure wetting layer problem or zero if the state is true QD state. Consequently Eq. (11) could be considered for 2 and 4 boundaries. Because it is presumed that boundary 2 and 4 are located at relatively large values of $r$, far from the QD region. The chosen values, shown in Figure 1. Satisfy this assumption.

It is strightfoward to mention that Eq. (7) is a partial diferentail equaion (PDE). We employ this fact that the presented formulate for QD system have to be investigated numerically. In this regard we developed our homemade codes using finite element method (FEM) to perform the numerical solution for the QD system with given boundary conditions. In our numerical approach we have considered trucated pyramids QD both in presence of wetting layer for $\mathrm{ZB}$ and $\mathrm{WZ}$ structure. The gremetry and dimension of the $\mathrm{GaN} / \mathrm{AlN}$ is considered according to Figure 1.

To investigate the optical properties we have considered a linear $x$-polarized electric field propagating along $z$ direction as:

$$
\tilde{E}(z, t)=E_{0} i e^{i(k z-\omega t)}+C C
$$

Where $\omega$ is the angular frequency, $k=\frac{N \omega}{c}$ is the propagation constant, $\mathrm{N}$ is the complex index of refraction consist of real and imaginary parts $\left(N=n_{R}+i n_{i}\right)$. By making use of wellknown nonlinear optics formalism the complex refractive index in the form of effective susceptibility will be as follows:

$$
N=\sqrt{1+\chi_{e f f}} \approx 1+\frac{1}{2} \chi_{e f f}
$$

Where the effective susceptibility is constructed from first, second and third order susceptibilities respectively and it is defines as:

$$
\chi_{e f f}=\chi^{1}+\chi^{2} \tilde{E}+\chi^{3} \tilde{E}^{2}
$$

The relative refractive index changes (RRIC's) and absorption 
coefficients ( $\left.\mathrm{AC}^{\prime} \mathrm{s}\right)$ are given by:

$$
\begin{aligned}
\frac{\Delta n}{n} & =\frac{1}{2} \operatorname{Re}\left(\frac{\chi_{e f f}}{n_{r}}\right)=\frac{1}{2 n_{r}} \operatorname{RE}\left(\chi^{1}+\chi^{2} \tilde{E}+\chi^{3} \tilde{E}^{2}\right) \\
& =\frac{\Delta n}{n}^{(1)}+\frac{\Delta n}{n}^{(2)}+\frac{\Delta n}{n}^{(3)}
\end{aligned}
$$

And

$$
\alpha=\frac{2 n_{i} \omega}{c}=\omega \sqrt{\mu / \epsilon_{R}} \operatorname{Im}\left(\epsilon_{0} \chi_{e f f}\right)=\alpha^{(1)}+\alpha^{(2)}+\alpha^{(3)}
$$

The susceptibilities are obtained by solving the density matrix method and perturbation theory in steady state for the QD system. The first, second, and third orders of susceptibility will be obtained as:

$$
\begin{aligned}
& \chi^{(1)}(\omega) \\
& =\frac{N}{\varepsilon_{0} \hbar}\left\{M_{21}^{2}\left[\frac{1}{\omega_{21}-\omega-i \gamma_{21}}+\frac{1}{\omega_{21}+\omega+i \gamma_{21}}\right]\right. \\
& \left.\quad+M_{31}^{2}\left[\frac{1}{\omega_{31}-\omega-i \gamma_{31}}+\frac{1}{\omega_{31}+\omega+i \gamma_{31}}\right]\right\} \\
& \chi^{(2)}(\omega)=\frac{N}{\varepsilon_{0} \hbar^{2}} \sum_{n, m=1}^{3} \mu_{1 n} \mu_{n m} \mu_{m 1} \\
& \left\{\frac{\left(\omega_{m 1}-i \gamma_{m 1}\right)\left[\left(\omega_{n 1}+\omega_{n m}\right)+i\left(\gamma_{n 1}-\gamma_{n m}\right)\right]}{\left[\left(\omega_{m 1}-i \gamma_{m 1}\right)^{2}-\omega^{2}\right]\left(\omega_{n 1}-i \gamma_{n 1}\right)\left(\omega_{n m}+i \gamma_{n m}\right)}\right. \\
& \left.+\frac{\left(\omega_{n 1}+i \gamma_{n 1}\right)\left[\left(\omega_{m 1}+\omega_{m n}\right)+i\left(\gamma_{m 1}-\gamma_{m n}\right)\right]}{\left[\left(\omega_{n 1}+i \gamma_{n 1}\right)^{2}-\omega^{2}\right]\left(\omega_{m 1}+i \gamma_{m 1}\right)\left(\omega_{m n}-i \gamma_{m n}\right)}\right\}
\end{aligned}
$$

And

$$
\begin{aligned}
& \chi^{3}=\frac{6 N}{\epsilon_{0} \hbar^{3}} \sum_{n m v=1}^{3} \mu_{1 v} \mu_{v n} \mu_{n m} \mu_{m 1} \\
& \left\{\frac{1}{\left[\left(\omega_{v 1}-\omega\right)-i \gamma_{v 1}\right]\left[\omega_{n 1}-i \gamma_{n 1}\right]\left[\left(\omega_{m 1}-\omega\right)-i \gamma_{m 1}\right]}\right. \\
& +\frac{1}{\left[\left(\omega_{n v}-\omega\right)-i \gamma_{n v}\right]} \\
& \cdot\left[\frac{1}{\left[\omega_{m v}-i \gamma_{m v}\right]\left[\left(\omega_{v 1}+\omega\right)+i \gamma_{v 1}\right]}\right. \\
& \left.+\frac{1}{\left[\omega_{v m}+i \gamma_{v m}\right]\left[\left(\omega_{m 1}-\omega\right)-i \gamma_{m 1}\right]}\right] \\
& +\frac{1}{\left[\left(\omega_{m n}-\omega\right)-i \gamma_{m n}\right]\left[\left(\omega_{n 1}\right)+i \gamma_{n 1}\right]\left[\left(\omega_{v 1}+\omega\right)+i \gamma_{v 1}\right]} \\
& +\frac{1}{\left[\left(\omega_{v n}+\omega\right)+i \gamma_{v n}\right]\left[\omega_{n 1}-i \gamma_{n 1}\right]\left[\left(\omega_{m 1}-\omega\right)-i \gamma_{m 1}\right]} \\
& +\frac{1}{\left[\left(\omega_{n m}+\omega\right)+i \gamma_{n m}\right]} \\
& \cdot\left[\frac{1}{\left[\omega_{m v}-i \gamma_{m v}\right]\left[\left(\omega_{v 1}+\omega\right)+i \gamma_{v 1}\right]}\right. \\
& \left.+\frac{1}{\left[\omega_{m v}+i \gamma_{m v}\right]\left[\left(\omega_{m 1}-\omega\right)-i \gamma_{m 1}\right]}\right] \\
& \left.+\frac{1}{\left[\left(\omega_{m 1}+\omega\right)+i \gamma_{m 1}\right]\left[\left(\omega_{n 1}\right)+i \gamma_{n 1}\right]\left[\left(\omega_{\nu 1}+\omega\right)+i \gamma_{\nu 1}\right]}\right\}
\end{aligned}
$$
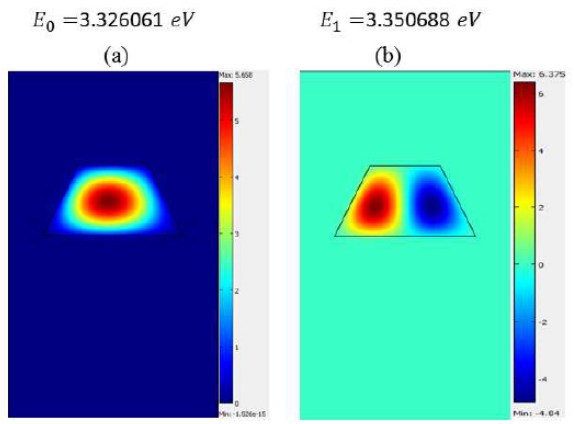

$E_{2}=3.374971 \mathrm{eV}$

(c)

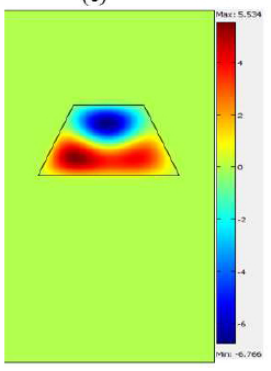

$E_{0}=3.31826 \mathrm{eV}$

(d)

$E_{1}=3.338322 \mathrm{eV}$

(e)
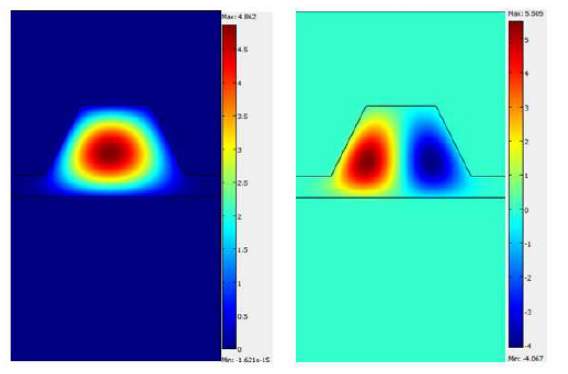

$E_{2}=3.348879 \mathrm{eV}$

(f)

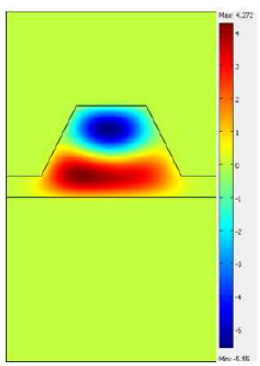

Fig 2

FIG. 2 The envelope function of GaN/AIN QDs (a) without wetting layer (b) with wetting layer (thickness $=3 \mathrm{~nm}$ ) plotted against $\mathrm{z}$ and $\mathrm{r}$ coordinates.

\section{RESULTS AND DISCUSSION}

The characteristics of GaN/AlN QDs are set according to Table 1 . To obtain the numerical results for different orders of susceptibility, the following parameters have been used: carrier density $N=3 \times 10^{22}$ atoms $/ \mathrm{cm}^{2}$ [38], vacuum permittivity $\varepsilon_{0}=8.85 \times 10^{-12}$, relaxation rate $\gamma=5 \times 10^{12} \mathrm{~s}^{-1}$ [38], speed of light in vacuum $c=3 \times 10^{8}$, transition frequencies $\hbar \omega_{21}=E_{2}-E_{1}$ (as output of numerical simulation), and transition dipole moments $\mu_{j i}=\left\langle\psi_{j}|-e r| \psi_{i}\right\rangle$ (e.g. $\left.\mu_{21}=\left\langle\psi_{2}|-e r| \psi_{1}\right\rangle\right)$ which is calculated by using numerical envelope function and homemade codes of Simpson integration method which is written in Matlab.

As it is mentioned previously, Figure 1 shows the geometry and the dimension of GaN/AlN QDs in the modeling procedure.

Figure 2 shows the envelope function for 3 states below the potential barrier is considered. These three subbands do not represent three different subbands in the usual sense, i.e. corresponding to 1,2 , and 3 nodes along the $\mathrm{z}$ direction. Figure 2(a)-(c) and Figure 2(d)-(f) illustrate the GaN/AIN QDs without and with wetting layer respectively. The thickness of wetting layer is set as $3 \mathrm{~nm}$ [38]. The energy eigenvalues are shown above each state. The presence of wetting layer in all subbands reduces the amount of energy eigenvalue ranging from 0.01 to $0.03 \mathrm{eV}$. Moreover in presence of wetting layer, part of the envelope function shares with wetting layer however the general localization does changes. According to our expectation, the envelope function has the biggest value at the center of the QD while it decays exponentially to zero for both 


\begin{tabular}{|l|l|l|l|l|}
\hline & GaN & AlN & \\
\hline Phase & wurtzite & zinc blende & wurtzite & zinc blende \\
\hline effective mass $\left(m_{\text {electron }}\right.$ & $0.20 \pm 0.02[39]$ & $0.193[40]$ & $0.29-0.45[41]$ & $0.316[40]$ \\
\hline band gap $(\mathrm{eV})$ & $3.50[39]$ & $3.3[42]-[44]$ & $6.0-6.3[45]$ & $5.34[46]$ \\
\hline
\end{tabular}

TABLE 1 Physical paramters Wurtzite and Zinc Blende GaN/AIN Quantum dots.
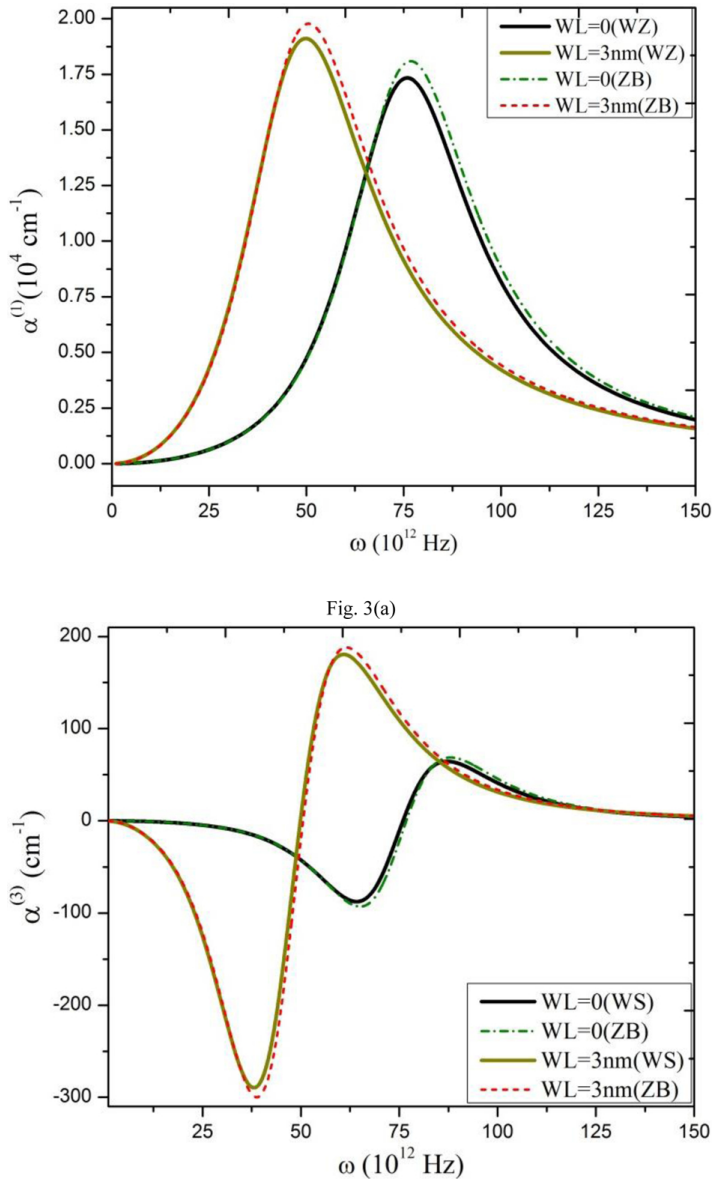

Fig. 3(b)

FIG. 3 The (a) first order, (b) third order absorption coefficients for ZB and WZ GN/AIN QDs with (solid lines) and without wetting layer (dashed and dash-dotted lines). The size of wetting layer set as $3 \mathrm{~nm}$ and the relaxation rates chosen to be $\gamma_{12}=\gamma_{13}=$ $\gamma_{23}=\gamma=5 \times 10^{12}$ and $\gamma_{11}=\gamma_{22}=\gamma_{33}=2 \gamma$ for all curves. For ZB case, the dipole moments without wetting layer are as calculated as $\mu_{12}=\mu_{21}=0.0026 \times$ $10^{-26}, \mu_{13}=\mu_{31}=0.2857 \times 10^{-27}, \mu_{23}=\mu_{32}=0.0002 \times 10^{-27}, \mu_{11}=0.493 \times$ $10^{-26}, \mu_{22}=0.486 \times 10^{-26}, \mu_{33}=0.4947 \times 10^{-26}$ and with wetting layer being $\mu_{12}=\mu_{21}=0.0087 \times 10^{-26}, \mu_{13}=\mu_{31}=0.3775 \times 10^{-27}, \mu_{23}=\mu_{32}=0.0033 \times$ $10^{-27}, \mu_{11}=0.4666 \times 10^{-26}, \mu_{22}=0.4532 \times 10^{-26}, \mu_{33}=0.4723 \times 10^{-26}$. For WZ case, the dipole moments without wetting layer are calculated as $\mu_{12}=\mu_{21}=$ $0.0024 \times 10^{-26}, \mu_{13}=\mu_{31}=0.2814 \times 10^{-27}, \mu_{23}=\mu_{32}=0.0001 \times 10^{-27}, \mu_{11}=$ $0.493 \times 10^{-26}, \mu_{22}=0.4861 \times 10^{-26}, \mu_{33}=0.4947 \times 10^{-26}$ and with wetting layer being $\mu_{12}=\mu_{21}=0.0082 \times 10^{-26}, \mu_{13}=\mu_{31}=0.3742 \times 10^{-27}, \mu_{23}=\mu_{32}=$ $0.0034 \times 10^{-27}, \mu_{11}=0.4667 \times 10^{-26}, \mu_{22}=0.4534 \times 10^{-26}, \mu_{33}=0.4723 \times$ $10^{-26}$.

in presence and absence of wetting layer (Figure 2(a),(d)). The peak of envelop function for ground state without wetting layer is about $\max =5.658$ (Figure 2(a)) while in the presence of wetting layer it dramatically reduces to be $\max =4.862$. As Figure 2(b),(e) depict the first excited state consists of a valley and a peak. The amplitude of the peak and the valley without wetting layer is $\max =6.375$ and $\min =-4.84$ respectively while in presence of wetting layer these values change to be $\max =5.565$ and $\min =-4.067$ respectively. Also the amount of energy eigenvalue is about $0.02 \mathrm{eV}$ smaller in presence of wetting layer. Figure 2(c),(f) shows the second exited state. This state has one valley on the top and a doublet peaks on bottom. The amount of the peak and valley is about $\max =5.34$ and $\min =-6.766$ in absence of wetting layer as Figure 2(c) depicts. Figure 2(f) shows the second excited state in presence of wetting layer. The significant difference appears for the doublet bottom peaks where they merge to construct a single peak which is mainly located at the left side of the QD. The amount of the peak and valley is about $\max =4.272$ and $\min =-5.55$. To be more precise, Figure 2(f) reveals that the right peak moves toward the left side in presence of wetting layer, as the red dark area in Figure 2(f) shows that the concentration of envelope function move to left side. Generally the envelop function in presence of wetting layer has bigger FWHM simultaneously the peaks takes smaller values.

We have used Eqs. (16) and (17), to investigate the optical properties of GaN/AlN QDs. Figure 3 shows the first and third order absorption for both ZB and WZ QDs with and without wetting layer. It is shown that there is a slight difference between $\mathrm{ZB}$ and $\mathrm{WZ}$ in linear and nonlinear absorption while the ZB has a slightly bigger value. Figure 3(a) shows that linear absorption profile is mainly consists of on dominant peak, while as Figure 3(b) shows the third order nonlinear absorption consist of a valley and peak, and most surprisingly it has a comparable value to linear absorption. Also it consists of a peak and valley unlike the linear absorption which has only one peak. The presence of wetting layer causes the peak to happen in lower frequencies more ever always a remarkable escalation occurs in the peaks. It is worthwhile to mention that a second order susceptibility has no contribution on the absorption profile. (e.g. it is completely real hence there is no second order absorption)

Figure 4 shows the first, second and third order RRIC's for GaN/Aln QDs. Figure 4(a) shows that linear RRIC without wetting layer begins with 0.01 value while in presence of wetting layer it begins with almost 0.025 . In the presence of wetting layer for both $\mathrm{WZ}$ and $\mathrm{ZB}$ structures the drop on the RRIC profile occurs in lower frequencies. However both curves come to steady state in same frequency. Figure 4(b) shows the RRIC due to second order nonlinear susceptibility. In presence of wetting layer the peak of second order RRIC takes almost 5 times larger than those without wetting layer. Figure 4(c) illustrates the third order RRIC which peculiarly has relatively stronger values in comparison to second order RRIC but is same order. Furthermore it is exhibited in Figure 4(c) that the RRIC has negative value initially and it rises to get a peak with positive value. In presence of wetting layer the RRIC is about 3.5 times larger than those of without wet- 


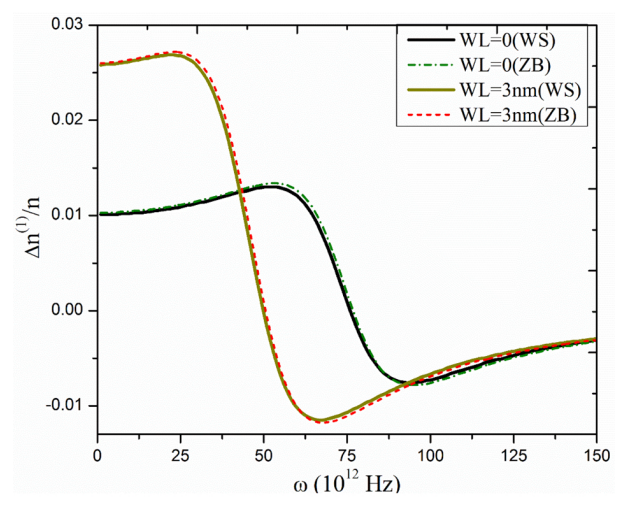

Fig. 4(a)

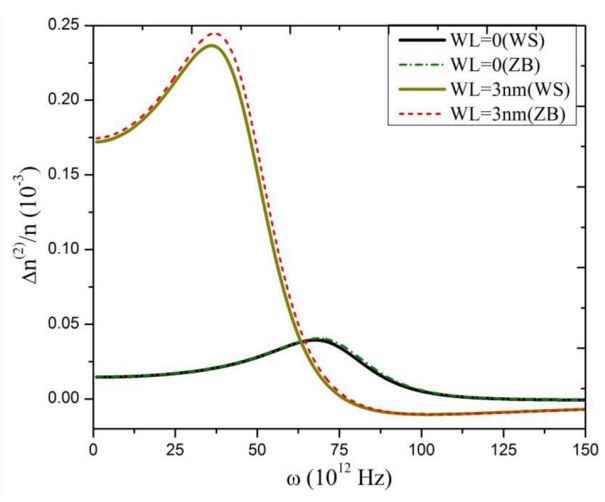

Fig. 4(b)

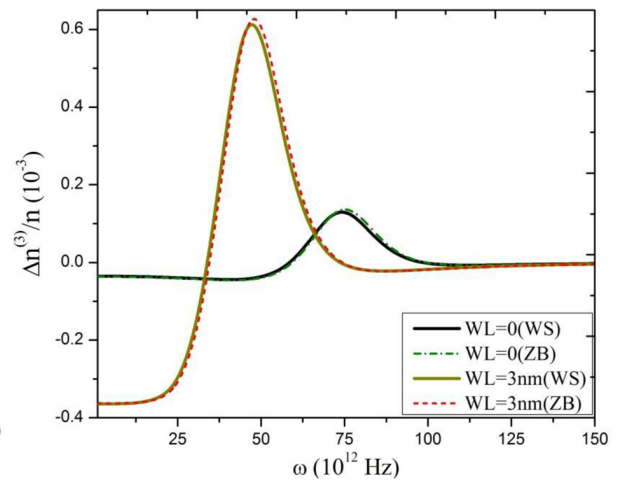

Fig. 4(c)

FIG. 4 The (a) first (b) second, and (c) third order relative refractive index changes for ZB and WZ GN/AIN QDs with (solid lines) and without wetting layer (dashed and dashdotted lines). The physical parameter set as Figure 3.
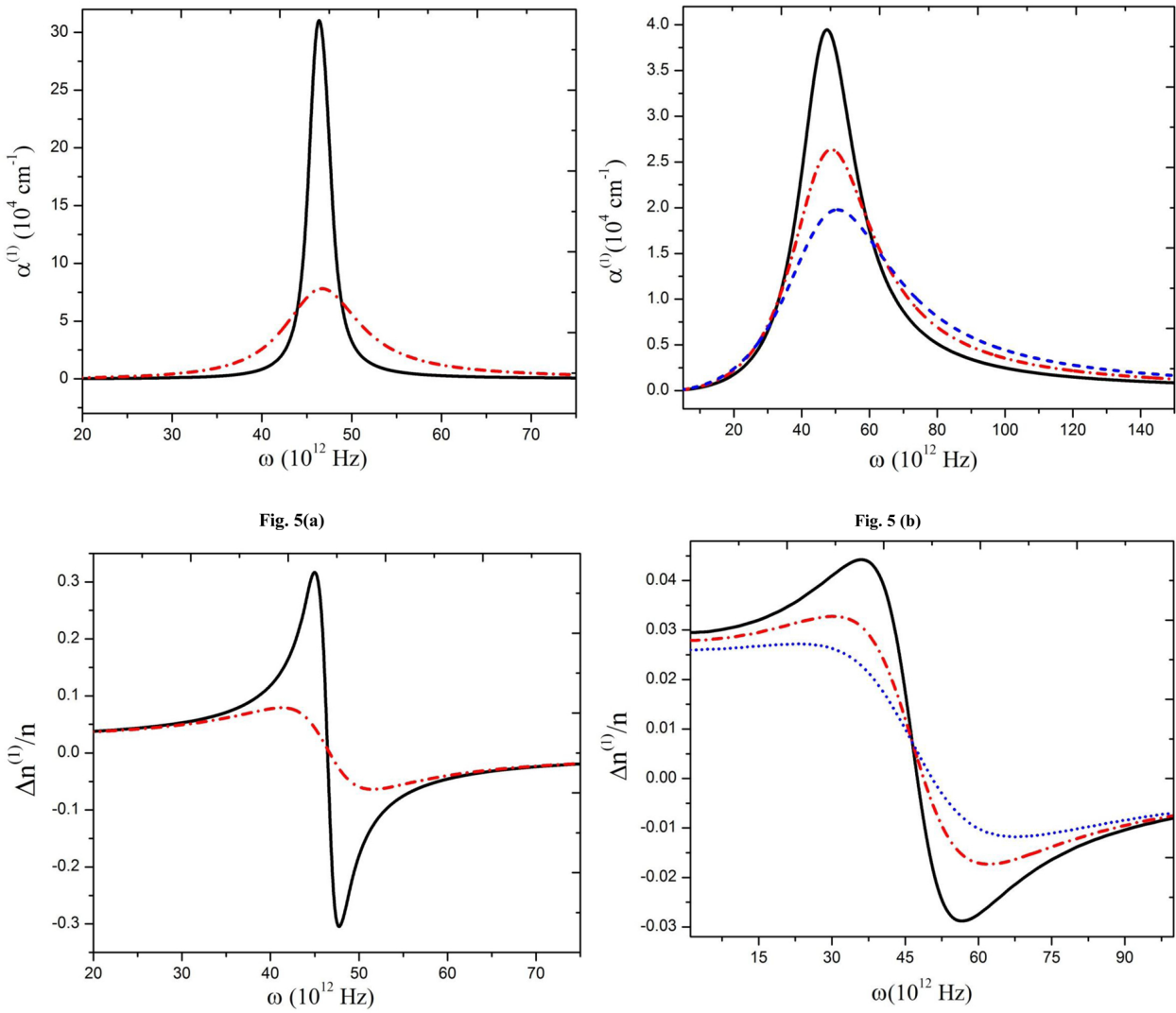

Fig. 5(C)

Fig. 5 (d)

FIG. 5 The first order optical properties of truncated pyramids GaN/AIN QDs for variant relaxation rates with wetting layer (thickness $=3 \mathrm{~nm}$ ). (a) First order absorption coefficient for $\gamma=1 \times 10^{12}$ (solid line), $\gamma=5 \times 10^{12}$ (dash-dotted line), and (b) $\gamma=10 \times 10^{12}$ (solid line), $\gamma=15 \times 10^{12}$ (dash-dotted line), and $\gamma=20 \times 10^{12}$ (dashed line), (c) first order relative refractive index changes for $\gamma=1 \times 10^{12}$ (solid line), $\gamma=5 \times 10^{12}$ (dash-dotted line), and (b) $\gamma=10 \times 10^{12}$ (solid line), $\gamma=15 \times 10^{12}$ (dash-dotted line), and $\gamma=20 \times 10^{12}$ (dashed line). Other physical parameters set as Figure 2 .

ting layer. Additionally the third order RRIC never becomes zero and always has slight negative value; even in relatively big frequencies, while first and second order become zero.

As the result until now shows there is a narrow different between WZ and ZB structures in favor of ZB QDs. Henceforward we chose to provide the result for $\mathrm{ZB}$ structures in subsequent curves, by virtue of that the result are quite predictable for WZ structures.

Figure 5 shows the linear absorption and RRIC for different amount of relaxation rates. In Figure 5(a),(c) for the aim of comparison, the $\gamma$ set as $1 \times 10^{12}$ (solid line) while it used to be $5 \times 10^{12}$ (dashed line). It is obvious that by reducing the amplitude of $\gamma$ to $1 / 5$ the values of the peaks depict 6 times larger inflation. Figure 5(c) shows that the RRIC similarly shows comparatively prodigious escalation for both peaks and valleys. Then to interrogate the effect of relaxation more deeply, its amount varied for 3 other values as it is shown in Figure $5(\mathrm{~b}),(\mathrm{d})$. These curves clearly shows that by rising the amount of relaxation rate the overall profile decreases both for linear absorption coefficient and RRIC. Afterward we investi- 


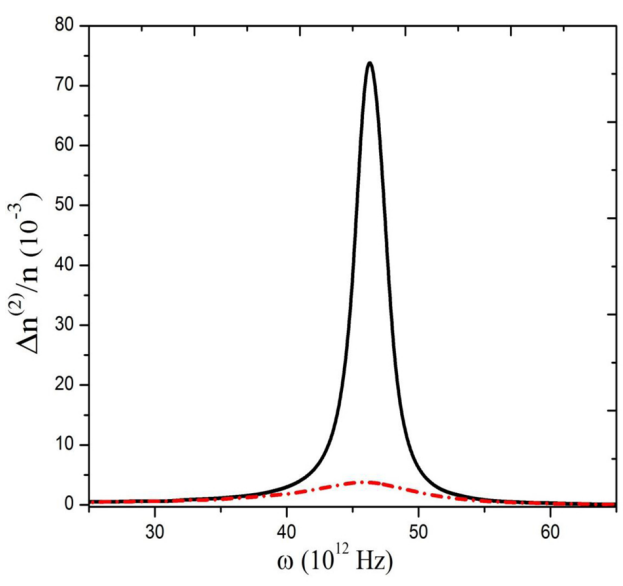

Fig.6 (a)

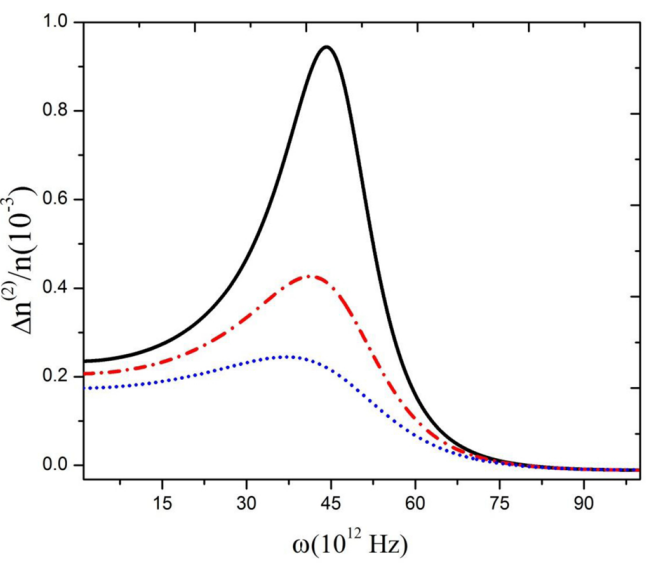

Fig. 6 (b)

FIG. 6 Second order refractive index changes (RRIC) versus the field probe frequency for different amount of relation rates for GaN/AIN with wetting layer. (a) Second order RRIC for $\gamma=1 \times 10^{12}$ (solid line), $\gamma=5 \times 10^{12}$ (dash-dotted line), and (b) $\gamma=10 \times 10^{12}$ (solid line), $\gamma=15 \times 10^{12}$ (dash-dotted line), and $\gamma=20 \times 10^{12}$ (dotted line). The physical parameters set as Figure 2 .
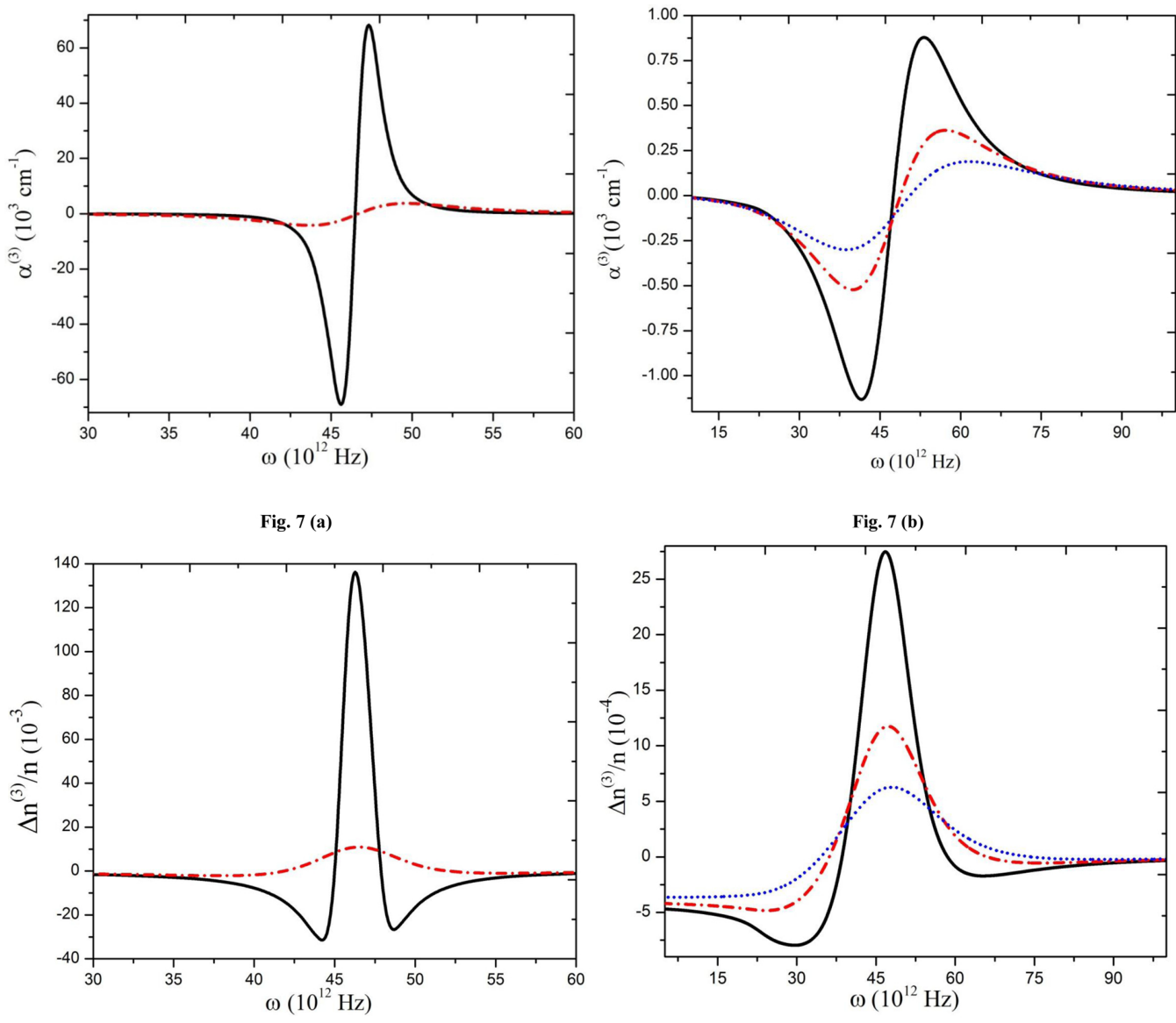

Fig. 7(C)

Fig. 7 (d)

FIG. 7 The third order optical property truncated pyramids GaN/AIN QDs for variant relaxation rates with wetting layer (thickness $=3 \mathrm{~nm}$ ). (a) First order absorption coefficient for $\gamma=1 \times 10^{12}$ (solid line), $\gamma=5 \times 10^{12}$ (dash-dotted line), and (b) $\gamma=10 \times 10^{12}$ (solid line), $\gamma=15 \times 10^{12}$ (dash-dotted line), and $\gamma=20 \times 10^{12}$ (dashed line), (c) first order relative refractive index changes for $\gamma=1 \times 10^{12}$ (solid line), $\gamma=5 \times 10^{12}$ (dash-dotted line), and (b) $\gamma=10 \times 10^{12}$ (solid line), $\gamma=15 \times 10^{12}$ (dash-dotted line), and $\gamma=20 \times 10^{12}$ (dashed line). Other physical parameters set as Figure 2 .

gate the variation of RRIC due to second order susceptibility as it is shown in Figure 6. Figure 6(a) shows that reducing the amplitude of $\gamma$ to1/5, the peak amplitude becomes about 60 times larger. Also as Figure 6(b) pageantries the effect of relaxation rate alteration is crucial however the bigger values remarkable decline take place. For instance the peak of RRIC for $\gamma / 5$ (Figure 6(a)-solid line) is about 80 times stronger than those for $2 \gamma$ (Figure 6(b)-solid line).

Figure 7 shows the third order absorption coefficient and RRIC for discussed system under the variation of relaxation rates. Figure 7(a) shows that when we reduce the amplitude 
of relaxation rate to $\gamma / 5$ the peak peaks of $\alpha^{3}$ shows an escalation with about 300 times (solid line) in comparison to that belong to $\gamma$ (dashed line). Similarly we applied same variation in relaxation rates for third order RRIC's. As Figure 7(c) shows the peak takes relatively 560 times larger amount for $\gamma / 5$, which is significant and noticeable. Afterward we have examined difference amount of relaxation rates as Figure 7(b) (for AC's) and Figure 7(d) (for RRIC's) illustrates. The overall dramatic reduction in the amount of peaks is demonstrated for higher amount of relaxation rates as it is clearly shown in Figure $7(\mathrm{c}),(\mathrm{d})$. However the FWHM which is bigger in smaller relaxation rates.

\section{CONCLUSION}

In this communication first of all we have discussed the modeling of GaN/AlN QDs. It is shown that the distribution of envelope function is affected by presence of wetting layer for all discussed subbands. Then by making use of numerical data we have calculated the linear and nonlinear optical properties of GaN $\backslash A I N$ QD. It is well demonstrated that there is a slight difference between the optical properties of WZ and ZB structures. It is well-proved that presence of wetting layer with $3 \mathrm{~nm}$ thickness causes around $10 \times 10^{12} \mathrm{~Hz}$ movement in the position of peaks in all optical properties of GaN/AIN QDs as well as significant escalation on the amount of peaks. Eventually we change the amount of relaxation rates. The variation of relaxation rates profoundly influences the whole optical properties. Although when we changed the relaxation rates, it is found that there are specific frequencies that they never show any variation. By proper selection of physical properties of QDs it is possible to engineer the point with constant properties. The presented result shows that GaN/AlN QDs are marvelous candidates for future applications in optical optoelectronic devices.

\section{ACKNOWLED GEMENTS}

A. Khaledi-Nasab and M. Rezaie would like to thank professors P. Lefebvre, G. Salviati for their helpful supports and invaluable helps.

\section{References}

[1] D. Williams, A. Andreev, and E. 0'Reilly, "Dependence of exciton energy on dot size in GaN/AIN quantum dots," Phys. Rev. B 73, 241301 (2006).

[2] R. Melnik, and M. Willatzen, "Bandstructures of conical quantum dots with wetting layers," Nanotechnology 15, 1 (2004).

[3] N. Akopian, N. Lindner, E. Poem, Y. Berlatzky, J. Avron, D. Gershoni, B. Gerardot, et al., "Entangled photon pairs from semiconductor quantum dots," Phys. Rev. Lett. 96, 130501 (2006).

[4] R. Stevenson, R. Young, P. Atkinson, K. Cooper, D. Ritchie, and A. Shields, "A semiconductor source of triggered entangled photon pairs," Nature 439, 179-182 (2006).

[5] R. Horn, P. Abolghasem, B. J. Bijlani, D. Kang, A. Helmy, and G. Weihs, "Monolithic source of photon pairs," Phys. Rev. Lett. 108, 153605 (2012).
[6] G. Juska, V. Dimastrodonato, L. 0. Mereni, A. Gocalinska, and E. Pelucchi, "Towards quantum-dot arrays of entangled photon emitters," Nat. Photonics 7, 527-531 (2013).

[7] J. Claudon, J. Bleuse, N. S. Malik, M. Bazin, P. Jaffrennou, N. Gregersen, C. Sauvan, et al., "A highly efficient single-photon source based on a quantum dot in a photonic nanowire," Nat. Photonics 4, 174-177 (2010).

[8] E. A. Stinaff, M. Scheibner, A. S. Bracker, I. V. Ponomarev, V. L. Korenev, M. E. Ware, M. F. Doty, et al., "Optical signatures of coupled quantum dots," Science 311, 636-639 (2006).

[9] S. Michael, W. W. Chow, and H. C. Schneider, "Group-velocity slowdown in a double quantum dot molecule," Phys. Rev. B 88, 125305 (2013).

[10] S. Ramanathan, G. Petersen, K. Wijesundara, R. Thota, E. Stinaff, M. L. Kerfoot, M. Scheibner, et al., "Quantum-confined Stark effects in coupled InAs/GaAs quantum dots," Appl. Phys. Lett. 102, 213101 (2013).

[11] H. R. Hamedi, A. Khaledi-Nasab, and H. Ghaforyan, "Tunneling Control of Transmission Coefficient and Group Index in a Quantum Dot Nanostructure," Advances in Condensed Matter Physics 2014, 589415 (2014).

[12] A. Khaledi-Nasab, M. Sabaiean, M. Sahrai, and V. Fallahi, “Optical Rectification and Second Harmonic Generation on Quasi-Realistic InAs/GaAs Quantum Dots: With Attention to Wetting Layer Effect," ISRN Condensed Matter Physics 2013, 530259 (2013).

[13] A. Khaledi-Nasab, M. Sabaeian, M. Sahrai, V. Fallahi, and M. Mohammad-Rezaee, "The effect of Woods-Saxon potential on envelope function, intersubband dispersion curves and group velocity of InAs/GaAs quantum dots with wetting layer," Physica E (2014) article in press.

[14] F. Ponce, and D. Bour, "Nitride-based semiconductors for blue and green light-emitting devices," Nature 386, 351-359 (1997).

[15] S. Lazar, C. Hébert, and H. Zandbergen, "Investigation of hexagonal and cubic GaN by high-resolution electron energy-loss spectroscopy and density functional theory," Ultramicroscopy 98, 249257 (2004).

[16] F. Widmann, B. Daudin, G. Feuillet, Y. Samson, J. Rouviere, and N. Pelekanos, "Growth kinetics and optical properties of selforganized GaN quantum dots," J. Appl. Phys. 83, 7618-7624 (1998).

[17] J. Chen, A. Markus, A. Fiore, U. Oesterle, R. Stanley, J. Carlin, R. Houdre, et al., "Tuning InAs/GaAs quantum dot properties under Stranski-Krastanov growth mode for $1.3 \mu \mathrm{m}$ applications," J. Appl. Phys. 91, 6710-6716 (2002).

[18] C. Reaves, R. Pelzel, G. Hsueh, W. Weinberg, and S. DenBaars, "Formation of self-assembled InP islands on a GalnP/GaAs (311) A surface," Appl. Phys. Lett. 69, 3878-3880 (1996).

[19] M. Hanke, M. Schmidbauer, D. Grigoriev, H. Raidt, P. Schäfer, R. Köhler, A.-K. Gerlitzke, et al., "SiGe/Si (001) Stranski-Krastanow islands by liquid-phase epitaxy: Diffuse $x$-ray scattering versus growth observations," Phys. Rev. B 69, 075317 (2004).

[20] S. Kako, K. Hoshino, S. Iwamoto, S. Ishida, and Y. Arakawa, "Exciton and biexciton luminescence from single hexagonal GaNất AIN self-assembled quantum dots," Appl. Phys. Lett. 85, 64 (2004).

[21] S. Prabhakar, and R. Melnik, "Influence of electromechanical effects and wetting layers on band structures of AIN/GaN quantum dots and spin control," J. Appl. Phys. 108,064330-064337 (2010).

[22] K. Kawasaki, D. Yamazaki, A. Kinoshita, H. Hirayama, K. Tsutsui, and Y. Aoyagi, "GaN quantum-dot formation by self-assembling droplet epitaxy and application to single-electron transistors," 
Appl. Phys. Lett. 79, 2243-2245 (2001).

[23] K. Hoshino, and Y. Arakawa, "UV photoluminescence from GaN self-assembled quantum dots on AlxGa1âĂß̧XN surfaces grown by metalorganic chemical vapor deposition," Phys. Status Solidi C 1 , 2516-2519 (2004).

[24] M. Razeghi, and A. Rogalski, "Semiconductor ultraviolet detectors," J. Appl. Phys. 79, 7433-7473 (1996).

[25] R. Dingle, D. Sell, S. Stokowski, and M. Ilegems, "Absorption, reflectance, and luminescence of GaN epitaxial layers," Phys. Rev. B 4, 1211 (1971).

[26] W. Yim, E. Stofko, P. Zanzucchi, J. Pankove, M. Ettenberg, and S. Gilbert, "Epitaxially grown AIN and its optical band gap," J. Appl. Phys. 44, 292-296 (1973).

[27] B. Monemar, "Fundamental energy gap of GaN from photoluminescence excitation spectra," Phys. Rev. B 10, 676 (1974).

[28] P. Lefebvre, and B. Gayral, "Optical properties of GaN/AIN quantum dots," C. R. Phys. 9, 816-829 (2008).

[29] L. S. Dang, G. Fishman, H. Mariette, C. Adelmann, E. MartinezGuerrero, J. Simon, B. Daudin, et al., "GaN quantum dots: Physics and applications," J. Korean Phys. Soc. 42, 657-661 (2003).

[30] G. Salviati, F. Rossi, N. Armani, V. Grillo, 0. Martinez, A. Vinattieri, B. Damilano, et al., "Optical and structural characterization of selforganized stacked GaN/AIN quantum dots," J. Phys.-Condens. Mat. 16, 115 (2004).

[31] J. Renard, R. Songmuang, C. Bougerol, B. Daudin, and B. Gayral, "Exciton and biexciton luminescence from single GaN/AIN quantum dots in nanowires," Nano Lett. 8, 2092-2096 (2008).

[32] V. A. Fonoberov, and A. A. Balandin, "Optical properties of wurtzite and zinc-blende GaN/AlN quantum dots," J. Vac. Sci. Technol. B 22, 2190-2194 (2004).

[33] N. Skoulidis, V. Vargiamidis, and H. Polatoglou, "Study of the structural and optical properties of GaN/AIN quantum dot superlattices," Superlattice. Microst. 40, 432-439 (2006).

[34] N. Nuntawong, S. Birudavolu, C. Hains, S. Huang, H. Xu, and D. Huffaker, "Effect of strain-compensation in stacked equation> 1.3 span style=," Appl. Phys. Lett. 85, 3050-3052 (2004).

[35] N. Nuntawong, J. Tatebayashi, P. Wong, and D. Huffaker, "Localized strain reduction in strain-compensated InAs/CaAs stacked quantum dot structures," Appl. Phys. Lett. 90, 163121 (2007).
[36] H. Zhao, R. A. Arif, Y.-K. Ee, and N. Tansu, "Self-consistent analysis of strain-compensated InGaNâĂşAIGaN quantum wells for lasers and light-emitting diodes," IEEE J. Quant. Electron. 45, 66-78 (2009).

[37] S. H. Park, Y. T. Moon, J. S. Lee, H. K. Kwon, J. S. Park, and D. Ahn, "Spontaneous emission rate of green strain-compensated InGaN/InGaN LEDs using InGaN substrate," Phys. Status Solidi A 208, 195-198 (2011).

[38] M. Sabaeian, and A. Khaledi-Nasab, "Size-dependent intersubband optical properties of dome-shaped InAs/CaAs quantum dots with wetting layer," Appl. Optics 51, 4176-4185 (2012).

[39] I. Vurgaftman, and J. Meyer, "Band parameters for nitrogencontaining semiconductors," J. Appl. Phys. 94, 3675-3696 (2003).

[40] P. Rinke, M. Winkelnkemper, A. Qteish, D. Bimberg, J. Neugebauer, and M. Scheffler, "Consistent set of band parameters for the group-III nitrides AIN, GaN, and InN," Phys. Rev. B 77, 075202 (2008).

[41] E. Silveira, J. Freitas, M. Kneissl, D. Treat, N. Johnson, G. Slack, and L. Schowalter, "Near-bandedge cathodoluminescence of an AlN homoepitaxial film," Appl. Phys. Lett. 84, 3501-3503 (2004).

[42] Z. Sitar, M. Paisley, B. Yan, R. Davis, J. Ruan, and J. Choyke, "AIN/GaN superlattices grown by gas source molecular beam epitaxy," Thin Solid Films 200, 311-320 (1991).

[43] G. Ramirez-Flores, H. Navarro-Contreras, A. Lastras-Martinez, R. Powell, and J. Greene, "Temperature-dependent optical band gap of the metastable zinc-blende structure $\beta$-GaN," Phys. Rev. B 50, 8433 (1994).

[44] H. Okumura, S. Yoshida, and T. Okahisa, "Optical properties near the band gap on hexagonal and cubic GaN," Appl. Phys. Lett. 64, 2997-2999 (1994).

[45] L. Chen, B. Skromme, R. Dalmau, R. Schlesser, Z. Sitar, C. Chen, W. Sun, et al., "Band-edge exciton states in AlN single crystals and epitaxial layers," Appl. Phys. Lett. 85, 4334-4336 (2004).

[46] M. P. Thompson, G. W. Auner, T. S. Zheleva, K. A. Jones, S. J. Simko, and J. N. Hilfiker, "Deposition factors and band gap of zinc-blende AlN," J. Appl. Phys. 89, 3331-3336 (2001). 